\author{
6. Teil: \\ Richterliche Ethik als Limitierung justiziellen Machtzuwachses
}

Ion Copoeru*

\title{
Gibt es überhaupt Raum für Ethik? Implementation richterlicher und staatsanwaltschaftlicher Diskursethik in Rumänien
}

\section{Politischer Hintergrund der Schaffung eines richterlichen Verhaltenskodex}

Die politischen Entwicklungen in Rumänien bieten das Bild eines Gemeinwesens im Übergang von einem kommunistischen, totalitären Regime zu einer europäisch gefärbten Demokratie. Der Verhaltenskodex für Richter und Staatsanwälte in Rumänien ( $D e$ ontological Code of Judges and Prosecutors (DCJP)), der erst 2005 als Teil der für den Beitritt zur EU vorgeschriebenen politischen Maßnahmen angenommen wurde, ist sowohl ein Ergebnis dieses Wandels als auch Zeichen seines Umfangs. Das Projekt zur Umsetzung und Verbesserung des DCJP ist das erste gemeinsame Forschungsprojekt des Obersten Verwaltungsrats in Rumänien und der akademischen Institutionen, die nicht am Netzwerk zur Aus- und Weiterbildung von Richtern teilnehmen. Es stellte gleichermaßen das erste Projekt im Rahmen des transdisziplinären »ProEtica «-Seminars $^{1}$ dar, das einen hohen interaktiven und anwendungsorientierten Charakter hat. ${ }^{2}$

* Der Beitrag ist bearbeitet worden von Ion Copoeru und Alexandrina Rãdulescu, wurde aber auf dem Symposium allein von Ion Copoeru vorgetragen.

1 ProEtica ist eine Gruppe akademischer Forscher und Vertreter verschiedener Berufsstände. Sie bemüht sich hauptsächlich um Aus- und Weiterbildung, Sensibilisierung für dieses Thema und die verstärkte Mitarbeit der Vertreter der verschiedenen Berufsstände bei der Selbstverwaltung ihres Berufsstandes. Die in dieser Gruppe geleistete Forschung umfasst den Bereich der Berufsethik und konzentriert sich auf die Förderung der politischen Maßnahmen und die Verbesserung der Qualität von Arbeitsabläufen in unterschiedlichen Berufsfeldern. Sie integriert Fähigkeiten und Fertigkeiten aus verschiedenen akademischen Disziplinen (Ethik, Politikwissenschaften, Organisationspsychologie, öffentliche Verwaltung, Rechtswissenschaften, Geschichtswissenschaften usw.) und verschiedenen Arbeitsbereichen. ProEtica hat mit Nichtregierungsorganisationen und anderen Interessenvertretern zusammengearbeitet, um die Kommunikation innerhalb und zwischen verschiedenen Berufsgruppen, wie auch öffentliche Diskussionen und Debatten zu fördern. Ihre Experten haben den Berufsorganisationen, die sich um eine verstärkte Mitarbeit ihrer Mitglieder bei der Gestaltung der Politik im Bereich ihrer Arbeitsfelder und im politischen Leben Rumäniens bemühen, logistische und theoretische Unterstützung zur Verfügung gestellt.

2 Dieses Projekt wurde ermöglicht durch die finanzielle Unterstützung des Nationalen Rates für wissenschaftliche Forschung im Hochschulbereich und wurde durchgeführt mit logistischer Unterstützung des Obersten Richterrates. Das Projekt profitierte von der engen und fruchtbaren Zusammenarbeit mit Dr. Sofia Chiricã, Expertin für Organisationspsychologie, Koordinatorin des Projekts zum neuen moralischen und beruflichen Profil des Richters in Rumänien. 
Es wurde der Versuch unternommen, das Prinzip der staatlichen Gewaltenteilung zu implementieren und eine wirksame Kontrolle der Institutionen des Staates durch die Justiz einzuführen. Aufgrund dessen nahm die Reform des Richterberufs und seiner Ausübung eine Schlüsselrolle im Prozess des politischen Wandels ein und war ein Indikator für erzielte Fortschritte bei der Umsetzung. Die Betonung des moralischen Aspektes in der beruflichen Rechtspraxis kann als wichtiger Schritt im Prozess der Demokratisierung und als starker Anstoß für eine erneuerte Dynamik der gesellschaftlichen und politischen Kreativität gesehen werden.

\section{Gründe für die Krise der Justiz}

Unser Ausgangspunkt war die These, dass die Krise in der Justiz im heutigen Rumänien und die Schwierigkeiten im Zusammenhang mit den demokratischen Reformen maßgeblich auf die Unfähigkeit der Juristen zurückzuführen sind, die Regeln und Normen ihres Berufsstandes zu reflektieren, Meinungen untereinander und mit anderen Experten auszutauschen und sich an der Ausarbeitung dieser Regeln und Standards zu beteiligen. Allgemeiner gesagt kann man von der Notwendigkeit sprechen, die Fähigkeit des Einzelnen zu individuellem oder kollektivem Umgang mit Normen und Regeln wiederherzustellen, so dass dies zu einer Verbesserung ihres beruflichen Verhaltens führt. Die Analyse derzeitiger Praktiken von einem moralischen Standpunkt aus nicht von einem moralisierenden - hat gezeigt, dass der Grund dieser Blockade letztlich in einer Art moralischem Konformismus und fehlendem zivilen Engagement liegt. Dies zeigt sich auch in der Unfähigkeit des Berufsstandes, ihrem Handeln eine neue Bedeutung beizumessen und die soziokulturelle Welt kontinuierlich neu zu erschaffen, indem sie ihr eigenes Handeln und ihre Aussagen modifizieren und anpassen. ${ }^{3}$

\section{Ansatz der Untersuchung}

Diese Unfähigkeit, mit dem gesellschaftlichen Umfeld zu agieren, zeigt sich deutlich in der Diskrepanz zwischen Worten und Taten. Daher bestand der erste Schritt unseres Ansatzes darin, den beruflichen Akt (in diesem Fall den Akt richterlichen Urteilens) wieder auf seine (vergessene, verfälschte, falsch ausgelegte) Bedeutung zurückzuführen. In einem zweiten Schritt wurden die Rahmenbedingungen richterlichen Urteilens wieder in den Vordergrund gestellt, ${ }^{4}$ indem der Bezug auf den pragmatischen Kontext, die gesellschaftliche, kulturelle und kommunikative Umgebung wiederhergestellt wurde.

3 Wir stützen uns hier auf die phänomenologische Subjekttheorie, wie sie vor allem von Edmund Husserl und Alfred Schütz vertreten wird. Vgl. dazu folgende Publikation: Copoeru, Intersubjectivity: Between Doxa and Praxis in: Copoeru/Szabo (2004), Beyond Identity - Transformations of the Identity in a (Post)-Modern World, in der wir unser Verständnis von Gemeinschaft auf Konzepte wie »praktische Gemeinschaft des Willens« und »gemeinsame Veränderung « gründen.

4 Zum Themenfeld »Normen in ihrem jeweiligen Kontext« vgl.: Marc Maesschalck (2001), Normes et contextes - Les fondements d'une pragmatique contextuelle. 
Aus unserer Perspektive auf den juristischen Berufsstand war es wichtig, den Ansatz einer Selbst-Erneuerung des Berufsstands und seiner Berufsausübung vorzuziehen. Diese Perspektive sollte aber keinesfalls die positivistischen Annahmen legitimieren oder stützen, die dem Verhalten der Juristen im Rechtssystem zugrunde liegen. Ganz im Gegenteil, wir betrachteten das Projekt der Umsetzung und der Verbesserung des DCJP als Maßnahme, den exzessiven Formen des Legalismus ${ }^{5}$ entgegenzuwirken und den Bereich der richterlichen Praxis den universellen Prinzipien des Rechts und der Gerechtigkeit zu öffnen. Wir betrachteten es als einen Teil der Bemühungen, das rumänische Justizsystem zu reformieren. Es geht uns im Besonderen um die Implementierung eines neuen Systems von beruflichen und moralischen Beurteilung von Richtern. ${ }^{6}$

Dabei war uns von Anfang an klar, dass sich moralische Fragen im Rahmen von Berufspraktiken auf Verhaltensweisen beziehen, die sich über einen langen Zeitraum hinweg entwickelt haben, und dass es daher erforderlich sein wird, sich kontinuierlich mit ihnen auseinander zu setzen und sie sowohl als Individuum aber auch als Kollektiv zu reflektieren. Unser Schwerpunkt lag nicht auf dem »Endprodukt « als solchem (z.B. einem neuen Kodex), sondern darauf, wie sich Einzelpersonen und Organisationen der moralischen Fragen, denen sie gegenüberstehen, und des Entscheidungsmechanismus, der in kritischen Situationen greift, bewusst werden. Wir waren uns einig, dass die Entwicklung einer Berufskultur, die es ermöglicht, normative ethische Standards und Praktiken »von innen « zu erstellen und zu modifizieren, im Wesentlichen von der Möglichkeit und der Fähigkeit des Einzelnen abhängt, ethische Aspekte ihres Berufslebens zu reflektieren und zu diskutieren. Unser Ansatz kollidierte daher des Öfteren mit der derzeitig herrschenden Meinung, die sich im Großen und Ganzen auf die Anwendung bereits erarbeiteter Normen konzentriert.

Bei zahlreichen Diskussionsrunden, die im Rahmen des ProEtica-Seminars mit Vertretern aus den Berufsfeldern der Medizin, des Journalismus, der Architektur, der Sozialarbeit etc. stattfanden, mussten wir feststellen, dass diese dazu tendieren, sich gemeinsam gegen diejenigen zu stellen, die ihrer Meinung nach versuchen, ihnen Normen und Regeln »von außen« aufzuerlegen. Sie fühlen sich ihrer streng definierten und eng gefassten beruflichen Rolle sehr verbunden und neigen dazu, jedwede Intervention von außen als Bedrohung ihrer beruflichen Identität zu sehen. Aus diesem

5 Wir verstehen unter Legalismus das Versäumnis, das Gesetz als lebenden Organismus zu begreifen und den Widerwillen, über den gesetzlichen Wortlaut hinaus dessen Implikationen für die Gesellschaft als Ganze zu sehen. Dazu: Richard W. Leopold, Elihu Root and the Conservative Tradition, (Oscar Handlin ed., 1954), p. 19 (zitiert nach: Fred Zacharias, Lawyers as Gatekeepers, abrufbar unter: http://ssrn.com/abstract=591655).

6 Die oben genannten Aktivitäten sind auch Teil des gemeinsamen Forschungsprojekts »Normative Strategien und Praktiken in Rumänien während der Zeit vor und nach dem Beitritt zur EU« (2006-2008, Leiter Ion Copoeru). Das Projekt konzentrierte sich auf theoretische Aspekte und empirische Untersuchungen der Berufskultur und Kommunikation sowie die Bewertung der Reflektionsmethoden zur Verbesserung des professionellen Verhaltens. Eine Sammlung von Studien und Aufsätzen mit dem Titel »Moral Dilemmas and Autonomy « wurde 2007 veröffentlicht und eine weitere, »Ethics and professional Culture«, ist derzeit im Druck (beide koordiniert von Ion Copoeru und Nicoleta Szabo). 
Grund war eine Strategie wichtig, die in keinster Weise den Eindruck vermitteln sollte, dass wir als »Experten« im Besitz der allein gültigen Wahrheit wären. Wir haben deutlich gemacht, dass wir uns als Vermittler ${ }^{7}$ und als Unterstützer verstehen. Eine Ad-hoc-Ethik des Dialogs diente als allgemeiner theoretischer Rahmen und als praktisches Mittel zur Lösung der (andernfalls unvermeidlichen) Spannungen und Konflikte, die solche Themen immer wieder unter Richtern auslösen. Das Projektteam entschied daher, den bestehenden Verhaltenskodex einer Überprüfung aus Sicht der derzeit üblichen moralischen Praktiken und seiner Anwendbarkeit zu unterziehen. Zweitens wurde beschlossen, dass er nicht »von außen« oder »von oben« auferlegt werden kann, sondern von denjenigen erarbeitet werden sollte, die ihn befolgen müssen.

Diese Methode sollte eine Alternative zu den positivistischen Praktiken der Justiz aufzeigen und sich mit einer der verfassungsmäßigen Schwächen des rumänischen Gemeinwesens befassen, nämlich der Verbreitung autoritärer Mechanismen zur Erlangung eines gesellschaftlichen Konsenses und dem Widerstreben der Anerkennung von Unterschiede.

\section{Zielsetzungen, Methoden und Ergebnisse der Untersuchung}

\section{Erstes Ziel: Verstärkte Wahrnehmung des Verhaltenskodex}

Das erste Ziel des Projekts bestand in der verstärkten Wahrnehmung des DCJP und seiner derzeit üblichen Anwendung in der richterlichen Berufspraxis.

Die Methode zur Umsetzung des DCJP bestand im Grunde in einer Verstärkung der Interaktion zwischen den Vertretern der Justiz, genauer: der Mobilisierung all ihrer intellektuellen und motivationsbezogenen Ressourcen. Den Ausgangspunkt bildete eine Debatte über die Bestimmungen des DCJP in Verbindung mit den persönlichen Erfahrungen der Teilnehmer und die anschließende Um- bzw. Neuformulierung dieser Bestimmungen. Die Änderung der DCJP-Paragraphen gehörte jedoch gerade nicht zu den Zielen des Projekts. Der Text des DCJP sowie der Text des neu vorgestellten Kodex bildeten vielmehr den Vorwand, um das Einvernehmen über die Notwendigkeit eines stabilen ethischen Rahmens für den juristischen Berufsstand zu erzielen. Das Projekt konzentrierte sich auf das, was über den Text, d.h. über die sonst nicht hinterfragten Regeln hinausgeht, die professionelles Verhalten diktieren. Die Teilnehmer ließen sich zu einer naiven Verteidigung oder Kritik der einen oder anderen Bestimmung hinreißen, die philosophische Zielsetzung bestand aber vielmehr in der Wiedereinführung einer moralischen Reflexion des beruflichen Handelns und der Wiederherstellung von Bezügen zwischen dem Berufsstand und der Gesellschaft durch die explizite Offenlegung der allgemeinen Grundsätze unseres heutigen Zusammenlebens. Für das Projekt war es von großer Bedeutung, dass die Teilnehmer sich mit den Regeln, die ihren Berufsstand bestimmen, auseinandersetzen und sich bewusst machen,

7 Vgl.: Zygmunt Bauman (1987), Legislators and Interpreters - On Modernity, Post-Modernity and Intellectuals. 
was ihre Berufsgemeinschaft ausmacht und was die eigene Identität in der heutigen rumänischen Gesellschaft konstituiert.

Wir hielten es für ein sehr ermutigendes Ergebnis, dass sich die Richter zu allererst mit der Analyse und der Kritik des derzeitigen DCJP auf der Grundlage ihrer beruflichen und moralischen Erfahrungen beschäftigten. Insbesondere zwei Fragen schenkten sie besondere Aufmerksamkeit:

Art. 18 (2), bekräftigt durch Art. 19, der die Bedingungen und Voraussetzungen richterlicher Meinungsäußerung festlegt - im Hinblick auf die berufliche und moralische Integrität ihrer Kollegen;

Art. 16 (1), der die Rolle des Vorsitzenden des Gerichts bei der Handhabung von Ethikfragen unterstreicht und die Bedeutung der Gerichte insbesondere als Wahrer der Unabhängigkeit und Unparteilichkeit eines jeden Richters nennt. ${ }^{8}$

\section{Zweites Ziel: Schaffung einer neuen Organisationskultur}

Die zweite Zielsetzung des Projekts bestand in der Schaffung und Festigung eines für die moralische Reflexion und die Verbesserung der Qualität beruflichen Handelns günstigen organisatorischen Umfeldes.

Die Tatsache, dass die Richter sich mit voller Begeisterung der Erstellung eines neuen Ethikkodex für Richter (Code of Ethics for Judges (CEJ)) widmeten und neue ethische Berufseinrichtungen, wie Komitees für ethische Beratung, vorschlugen, ist sicherlich ein Schritt hin zur Schaffung und Pflege einer neuen Organisationskultur in der rumänischen Justiz. An dem Projekt konnte sich zwangsläufig nur eine geringe Anzahl von Teilnehmern (um die 50) beteiligen, aber es soll Modellcharakter für Debatten über moralische Fragen in der Justiz haben und zur Institutionalisierung des Dialogs im juristischen Berufsstand beitragen.

Obwohl das aus dem Seminar entstandene Gutachten durch den Obersten Rat der Magistratur gefördert wurde, hat der neue Ethikkodex für Richter nicht den älteren DCJP ersetzt. Berufsverbände der Richter haben es vorgezogen, ihre eigenen Verhaltenskodizes zu formulieren, die dem alten sehr ähneln. Der Vorschlag zur Einrichtung von Komitees für ethische Beratung auf Ebene der Berufungsgerichte blieb ebenfalls erfolglos. Kürzlich hat der Oberste Rat der Magistratur seine internen Regelungen geändert, damit er nun als eine Art nationaler Ethikrat fungieren kann. Einige Fälle sind diskutiert worden, aber es wurde deutlich, dass die Art und Weise, wie der Oberste Rat der Magistratur diese Fälle handhabt - in starker Anlehnung an Disziplinarverfahren für die Eigenheiten moralischer Fälle nicht geeignet ist.

8 Es wurden natürlich noch viele andere Themen diskutiert, wie die Aufgabe des Richters, das Verständnis von Moral, Unparteilichkeit und Unabhängigkeit des Richters und der Justiz, sowie die Rolle des Obersten Richterrates bei der Führung des Berufsstandes und der Sicherstellung der Unabhängigkeit der rumänischen Justiz. 


\section{Schlussfolgerungen}

Das große Interesse an Verhaltenskodizes ist sicherlich ein Zeichen dafür, dass eine ernsthafte Reflektion über die Aufgabe, die Werte und die Normen der Justiz in Rumänien stattfindet. Einige Fortschritte wurden bereits erzielt, viele weitere müssen noch folgen. An dieser Stelle des Veränderungsprozesses sind wir davon überzeugt, dass weitere Fortschritte von der Handhabung ethischer Fragen durch Richter abhängen.

\section{Bestehende Probleme}

Bisher haben die Vorsitzenden der Gerichte (auf lokaler Ebene) und der Oberste Rat der Magistratur (auf zentraler Ebene) diese Regeln angenommen und angewandt, ohne sie auf den gesamten Berufsstand auszuweiten oder eine tiefgreifende Betrachtung über die Besonderheiten der Ethik anzustoßen. Das führte auf beiden Ebenen zu einer Reihe von Schlüsselfragen. Der Vorsitzende des Gerichts erhält auf diese Weise zu viel Macht, übt diese paternalistisch aus und lässt dadurch Raum für willkürliche Strafen. Die in hohem Maße zentralisierten Verfahren des Obersten Rates der Magistratur sind für die Handhabung moralischer Fragen nicht geeignet: In den vergangenen Jahren sind sie beiseite geschoben und einfach nicht behandelt worden.

\section{Gründe für halbherzige Maßnahmen}

Sind halbherzige Maßnahmen in diesem Bereich der Preis für Zweckmäßigkeit oder ist dies der Hinweis auf ein tiefgreifenderes Problem?

Der DCJP ist unter dem Zeitdruck des Beitritts zur EU erarbeitet und eingeführt worden. Eine detaillierte Beratung fand nicht statt. Dieses Verfahren ist bedauerlicherweise charakteristisch für den politischen Führungsstil der Übergangszeit. Es erinnert uns an andere, nicht zu Ende geführte Modernisierungsversuche der letzten zwei Jahrhunderte. Ein veralteter paternalistischer und bürokratischer Entscheidungsfindungsprozess, gekennzeichnet durch eine Politik, in der Entscheidungen von oben auferlegt werden und die öffentliche Beratung begrenzt und instrumentalisiert wurde. Die überhandnehmende Konzentration auf die Anwendung von Disziplinarmaßnahmen harrt immer noch einer Änderung der Einstellung gegenüber Politik und Ethik als Kern und Stütze der Demokratisierungsbemühungen in Rumänien.

\section{Ausblick: Potential richterlicher Ethik als Grundlage für die anzustrebende Selbst- verwaltung der rumänischen Justiz}

Dennoch bleibt die Selbstverwaltung der rumänischen Justiz weiterhin das anzustrebende Ideal. Eine detaillierte Analyse einer Reihe von Berufsnormen ist in die Wege geleitet worden, und der Prozess ist heute irreversibel. Die Schwierigkeiten einer Kodifizierung richterlicher Ethik in der rumänischen Justiz zeigen die Grenzen eines übermäßig zentralisierten Systems und der damit verbundenen positivistischen Berufspraktiken. 
Die Unterscheidung von Ethik und juristischem Verfahren bildet wohl derzeit die wichtigste Frage in einer breiten normativen Debatte über Ethik in der Justiz. Hierbei geht es um die Konzeptualisierung von richterlicher Verantwortung. Sie muss verstanden werden als Zweigespann, bestehend aus Regeln, die die Rolle richterlicher Verantwortung im juristischen Prozess festlegen, und persönlicher Integrität. Zusammen können sie Regeln und Selbstverwaltung ihres Berufsstandes besser aufeinander abstimmen und den Bedürfnissen einer demokratischen Gesellschaft anpassen. Ethische Regeln dürfen nicht länger als persönliche Angelegenheit des Richters und daher als überflüssig für die Organisation in ihrer Gesamtheit gesehen werden. Im Gegenteil, sie ist von grundlegender Bedeutung für die Verwaltung einer Justiz, deren Ziel die Verwirklichung von Gerechtigkeit und Rechtsstaatlichkeit ist. 